\title{
Automatic Adjustment of Integration Time in the NIR Camera for Calibrated Temperature Measurement during Inductive Heating Processes
}

\author{
by Piotr Więcek, Jerzy Zgraja, Dominik Sankowski
}

Institute of Applied Computer Science, Lodz University of Technology, 90-924 Lodz, Stefanowskiego 18/22, Poland, pwiecek@gmail.com

\begin{abstract}
The system presented is based on a Near Infrared Radiation $(N I R)$ camera. The $C C D$ camera selected absorbs a small portion of radiation in the near infrared range, above $\lambda=700 \mathrm{~nm}$. In addition, by using an NIR filter one can cut off the visible light to reduce noise and unwanted radiation. The system has been developed at the Institute of Applied Computer Science, Lodz University of Technology. This system has been made for controlling industrial thermal processes during the inductive heating hardening. The varying of camera integration time has been applied to avoid the saturation and to increase the sensitivity at a low temperature. The emissivity of the inductive heating object has been taken into account. New algorithms for temperature calibration and image processing have been developed enabling the measurement of temperatures from $250^{\circ} \mathrm{C}$, thanks to the application of automatic adjustment of integration time.
\end{abstract}

\section{Introduction}

Using a Near Infrared Radiation spectral range to measure high temperatures is known in the literature [1-4]. High temperature object monitoring is the main application of such systems, e.g. in metallurgy [5], inductive heating [2] or for high temperature industrial processes [6]. Among many existing solutions using an NIR spectral range to measure high temperature objects, both monochrome and colour CCD camera-based systems are discussed [1,2]. All of them use a fixed integration time of the sensor. The previous systems allow the measurement of temperatures above $500^{\circ} \mathrm{C}$. In addition, the emissivity is not always taken into account. Some of them use a concept of multispectral radiation measurement [5].

The novel method of the calibration of a $C C D$-NIR camera is presented in this paper. The novelty of this method consists in automatic correction of the camera signal according to the integration time that is adjusted to the intensity of incoming radiation in the NIR spectrum. The approximation of the calibration curve by the exponential function was proposed and verified experimentally. The examples of the temperature measurements both in static and dynamic thermal processes are presented in this paper. The emissivity was consideration into account during calibration and measurement.

\section{CCD-NIR system with automatic adjustment of integration time}

The system presented is based on a broadband high-sensitivity 12-bit monochrome CCD camera. The camera is equipped with an NIR interferential filter that cuts off the visible range - fig. 1 . The spectral range of radiation absorption covers both VIS and NIR wavelength ranges up to $1000 \mathrm{~nm}$ - fig. 2. Special software was developed to vary the integration time according to the incoming light intensity. This means that at the beginning of heating, the high integration time is applied, while at the end of the temperature rise, the integration time is reduced to avoid the signal saturation. The spectral characteristics of the camera and the NIR filter are presented in fig. 2.

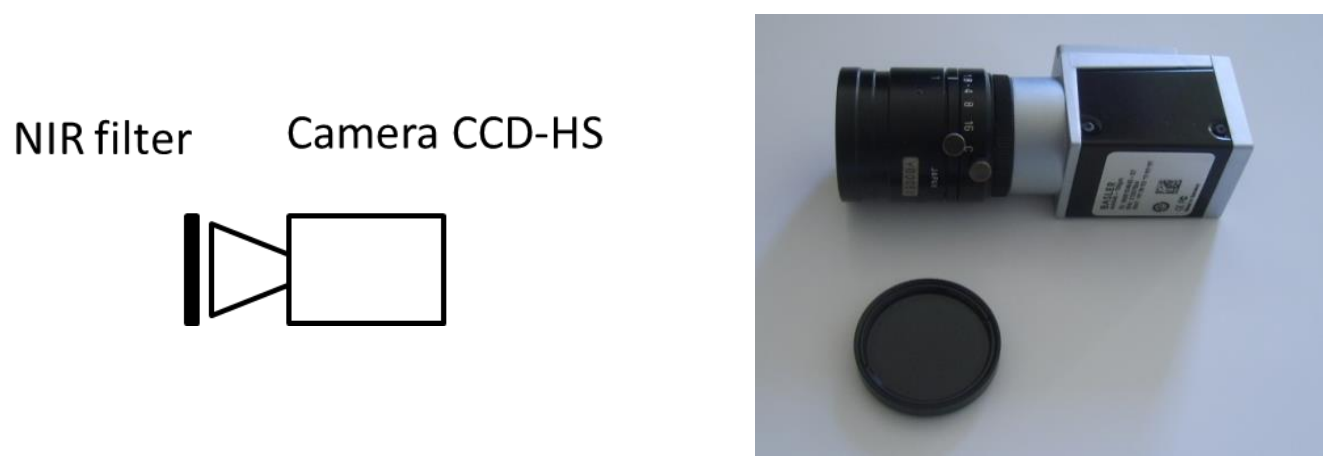

Fig. 1. High sensitivity CCD-HS with NIR filter 


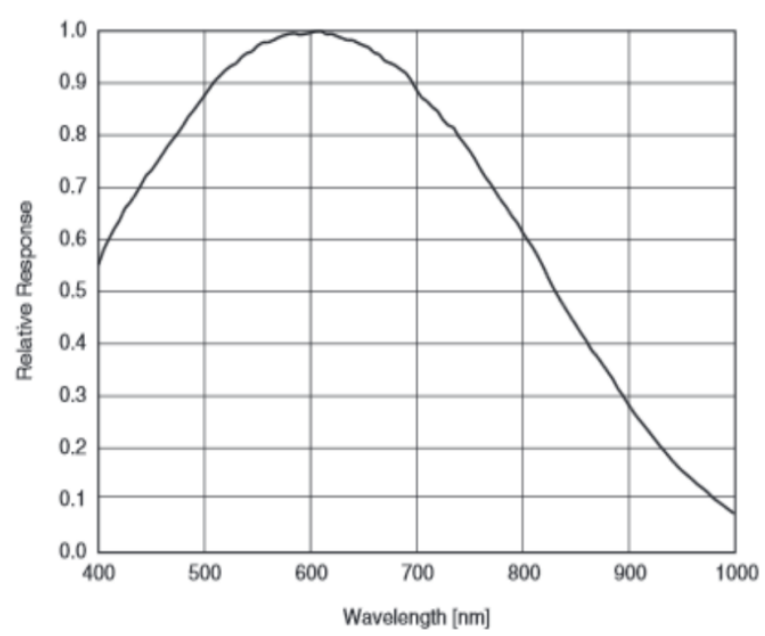

a)

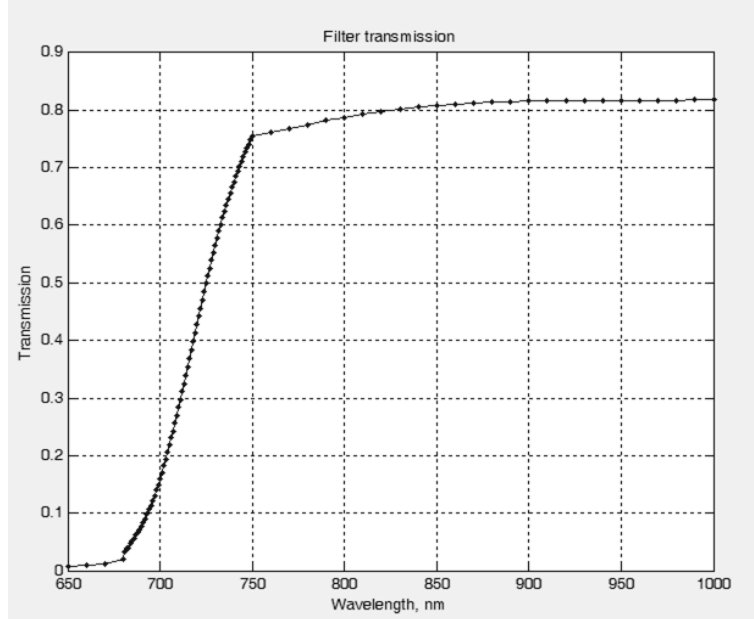

b)

Fig. 2. Spectral characteristics of a $C C D$ of high sensitivity (a) and an NIR filter (b)

Considering a large range of changes in radiation intensity and a high sensitivity of the $C C D$ camera, software for the registration of images with variable integration time $t_{\text {int }}$ was developed. To eliminate the effect of a change of integration time on the value of the digital signal DL (Digital Level) of the camera, i.e. to ensure the consistency of the DL/tint ratio, correction of the digital value of the signal generated by the camera $\left(D L_{c a m}\right)$ was introduced based on the following relationship:

$$
D L=D L_{\text {cam }} \frac{10^{6}}{t_{\text {int }}}
$$

where the integration time $t_{\text {int }}$ is expressed in $\mu \mathrm{s}$. The maximum integration time of the camera used in the investigations was $1 \mathrm{~s}\left(10^{6} \mu \mathrm{s}\right)$.

To confirm the correctness of use of relationship (1), a simple experiment was made. For a selected temperature of the object, a camera signal was determined at different integration times. The results are presented in Table 1.

Table 1. CCD-NIR camera signal for different integration times

\begin{tabular}{|c|c|c|c|c|c|c|c|c|}
\hline$t_{\text {int }, \mu \mathrm{S}}$ & $500=t_{\text {into }}$ & 600 & 700 & 800 & 900 & 1000 & 1100 & 1200 \\
\hline$D L$ & $1625=D L_{0}$ & 1963 & 2309 & 2695 & 3026 & 3327 & 3575 & 4015 \\
\hline
\end{tabular}

A chart for $D L / D L_{0}=\mathrm{f}\left(t_{\text {int }} / t_{\text {into }}\right)$ was made. As expected, this function is nearly linear, which confirms the correctness of use of the relationship (1), i.e. obtaining $D L / t_{\text {int }}=$ const - fig. 3 . The values $D L_{0}=1625$ and $t_{\text {into }}=500 \mu$ s denote the camera signal and integration time of the first measurement in Table 1.

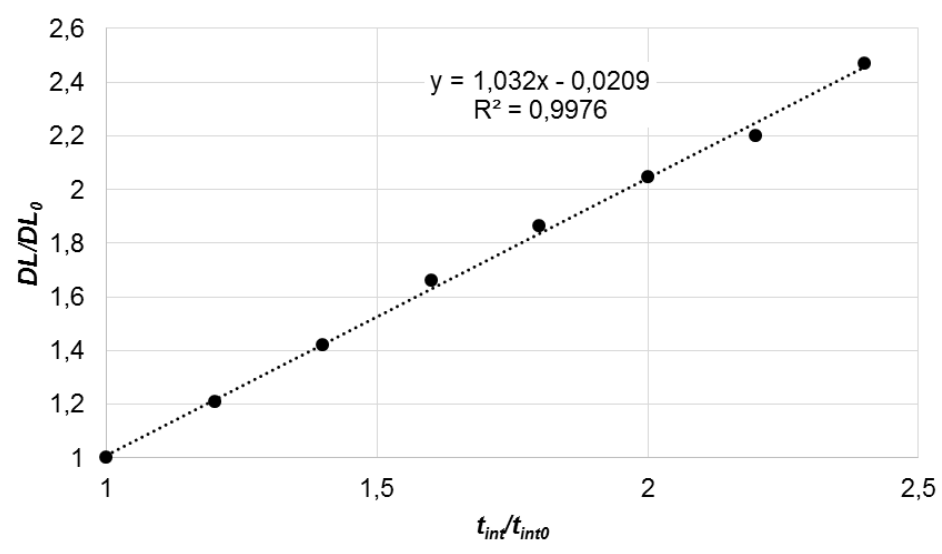

Fig. 3. Relationship $D L / D L_{0}=\mathrm{f}($ tint /tint $)$ 


\section{Method of calibration of a CCD-NIR camera using a black body for the MWIR and LWIR ranges}

\section{Determination of emissivity of a calibration object}

In the research a technical black body (calibration object) for spectral ranges MWIR (3-5 $\mu \mathrm{m})$ and $L$ WIR (8-12 $\mu \mathrm{m})$ was used. Within these ranges the emissivity of a black body $\varepsilon \approx 1$. Unfortunately, it is considerably lower for the $N I R$ range. The aim of the research was to determine the emissivity of the calibration object for the NIR range of approximately $700-1000 \mathrm{~nm}$. To this end a set of measurements was made.

Measurement 1 was carried out for different values of temperature of the calibration object in the range of 300$500^{\circ} \mathrm{C}$. The $C C D-N I R$ camera signal was determined under darkened conditions (without background radiation) - fig. 4 . It was assumed that in such conditions only the object radiates and its emissivity is $\varepsilon$.

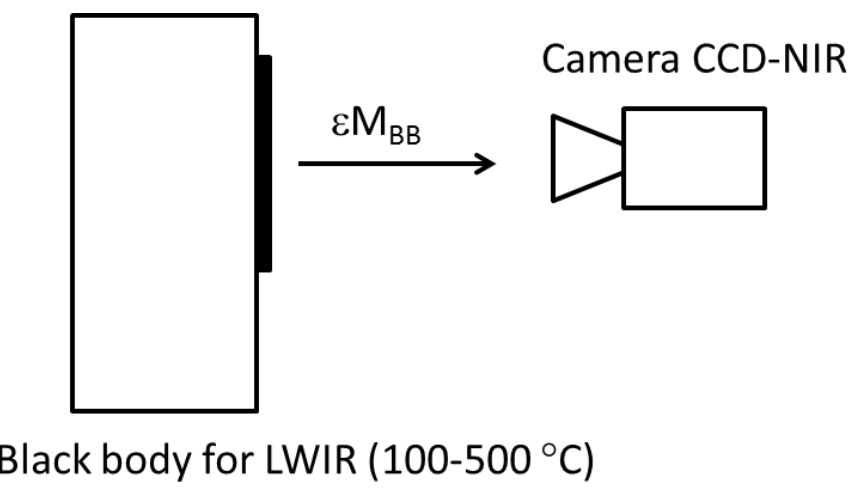

Fig. 4. Measurement of radiation intensity of the calibration object in the NIR range in darkened conditions $\left(D L_{o}\right)$

The second measurement was made in laboratory light conditions (sunlight/interior light). A stronger signal of measurement of radiation intensity was obtained due to the reflected background radiation - fig. 5 .

The third measurement dealt with background radiation. To this end, polished copper sheet was prepared which performed the function of a mirror in the NIR spectral range.

The background radiation intensity was measured and the measurement was made at room temperature $T_{a}=25^{\circ} \mathrm{C}-$ fig. 6 . To obtain the correct result of background radiation, the copper mirror must be placed in the same location as the reference object.

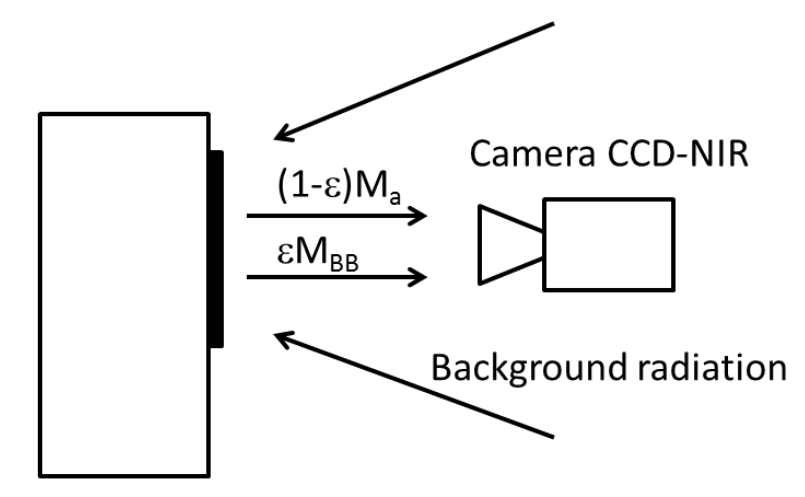

Black body for LWIR $\left(100-500^{\circ} \mathrm{C}\right)$

Fig. 5. Measurement of radiation intensity of the calibration object in the NIR range in laboratory conditions $(D L)$ 


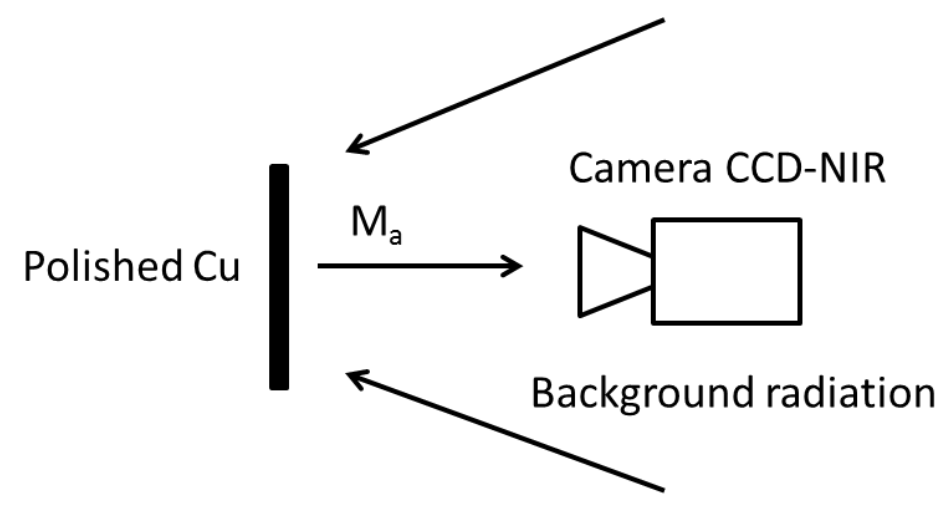

Ambient $\left(25^{\circ} \mathrm{C}\right)$

Fig. 6. Measurement of radiation intensity of the background in the NIR range in laboratory conditions $\left(D L_{a}\right)$

Basing on the measurement conditions presented in 4-6 the following relationships can be written (2)-(4).

$$
\begin{aligned}
& \varepsilon M_{B B}=A * D L_{o} \\
& (1-\varepsilon) M_{a}+\varepsilon M_{B B}=A * D L \\
& M_{a}=A * D L_{a}
\end{aligned}
$$

where MBB denotes the spectral luminous exitance of a black body radiation in the NIR range, Ma - the background radiation exitance, $A$ is the constant of the infrared camera.

Using equations (2)-(4) the emissivity of a calibration object can be determined, equations (5) and (6).

$$
\begin{gathered}
(1-\varepsilon) D L_{a}+D L_{o}=D L \\
\varepsilon=1-\frac{D L-D L_{o}}{D L_{a}}
\end{gathered}
$$

The results of the emissivity measurement of the calibration object for different temperature values are presented in Table 2. As can be seen from Table 2 the black body for MWIR and LWIR is not a black body for the NIR range.

Table 2. Emissivity of the calibration object

\begin{tabular}{|c|c|c|c|c|}
\hline $\begin{array}{c}\text { Temperature of the } \\
\text { calibration object } \\
\left({ }^{\circ} \mathrm{C}\right)\end{array}$ & $\begin{array}{c}\text { Darkening } \\
\left(D L_{o}\right)\end{array}$ & $\begin{array}{c}\text { Laboratory lighting } \\
(D L)\end{array}$ & $\begin{array}{c}\text { Reflection from the } \\
\text { mirror } \\
\left(D L_{a}\right)\end{array}$ & $\begin{array}{c}\text { Emissivity of the } \\
\text { black body } \\
(L W I R)\end{array}$ \\
\hline 250 & 26 & 662 & 11640 & 0.95 \\
\hline 300 & 101 & 815 & 12904 & 0.94 \\
\hline 350 & 684 & 1335 & 12264 & 0.95 \\
\hline 400 & 3870 & 4525 & 12192 & 0.95 \\
\hline 450 & 18130 & 18815 & 12360 & 0.94 \\
\hline
\end{tabular}

\section{Calibration of the CCD-NIR camera}

The measurements were made in the absence of background radiation, in a dark room with the use of an MWIR (3-5 $\mu \mathrm{m})$ infrared camera. The experiment was carried out according to fig. 7. Two cameras operating in parallel were used: a CCD-NIR and an MWIR infrared camera. The emissivity of the calibration object for the NIR range was taken into account in the measurements. The calibration curve assumes the shape of a logarithmic function - fig. 8 . When plotting the calibration curve, the conversion of digital values was taken into consideration according to the current value of the integration time according to relationship (1). Because of this conversion, the calibration curve does not depend on the integration time. The calibration curve was plotted based on areas of $16 \times 16$ pixels in the central section of images of both NIR and MWIR cameras. During the measurement, owing to the calibration curve determined one can calculate vales of 
temperature for each pixel of an image although due to the in-camera noise/proprietary noise of the camera, it is more appropriate to determine the temperature as a mean value for a number/several dozen of adjacent pixels.

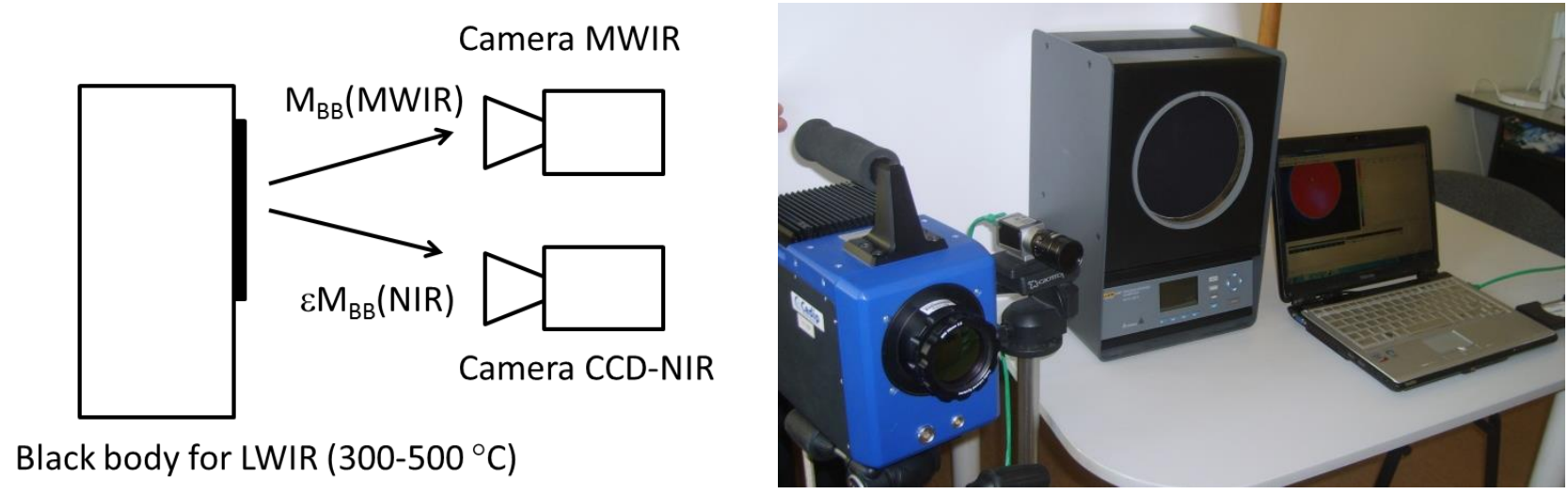

Fig. 7. Stand for the CCD-NIR camera calibration

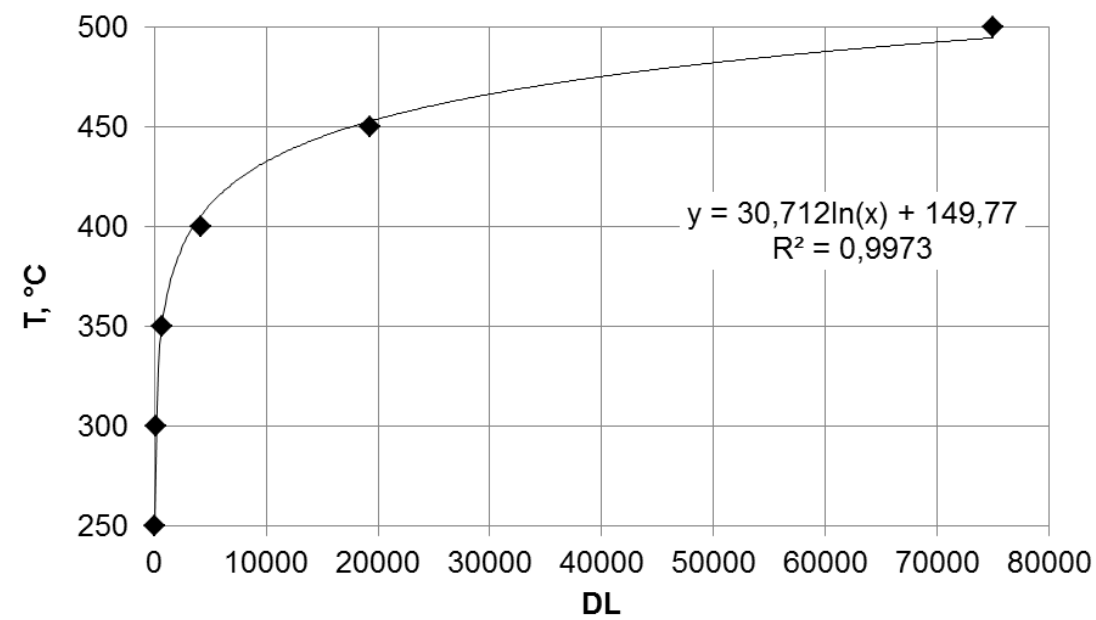

Fig. 8. Calibration curve for a range of $250-500^{\circ} \mathrm{C}$

\section{Measurement of temperature of an object of the emissivity $\varepsilon$}

Prior to measuring temperature, the emissivity of the object under study in the NIR range should be determined. This is done at room temperature for which the object's own radiation in the NIR range can be neglected. Two measurements must be made. The first one aims at determining the intensity of radiation reflected from the object of the emissivity $\varepsilon$ in the lighting conditions that will prevail during temperature measurement. As a result, the radiation intensity $D L$ is obtained. Then, the background radiation $D L_{a}$ is measured by means of a copper mirror - fig. 6. A very important parameter of this measurement is the positioning of the mirror in the place of the object studied (right in front of it) so that the mirror will reflect the same radiation as the object under study during the temperature measurement.

Equations (7) and (8) hold true for this experiment:

$$
\begin{aligned}
& (1-\varepsilon) D L_{a}=D L_{\varepsilon} \\
& \varepsilon=1-\frac{D L_{\varepsilon}}{D L_{a}}
\end{aligned}
$$

Once the object emissivity value is determined, we proceed to the proper measurement of the temperature value. This is done in the same conditions in which the emissivity was previously determined. We determine the object radiation intensity value $D L_{o}$ based on the measurement of intensity $D L$ under conditions of lighting of the object by background radiation - equation (9). 


$$
(1-\varepsilon) D L_{a}+\varepsilon D L_{o}=D L
$$

The measurement can be performed under darkened conditions. Then, the value of the background radiation should be assumed as $D L_{a}=0$. In such a case, the object radiation $D L_{o}=D L / \varepsilon$. Knowing the value $D L_{o}$ of the object, we apply the previously determined calibration curve for calculating the temperature value of the object (equation in fig. 8).

\section{Preliminary results}

The first results in the form of IR images are presented in fig. 9. We noticed that the developed system allowed one to measure the temperature above $250-300^{\circ} \mathrm{C}$. The images in fig. 9 were obtained with the fixed integration time. In addition, we could not get the signal below $300^{\circ} \mathrm{C}$ due to a relatively high noise. Another problem is the influence of the emissivity and ambient radiation on the temperature measurement. One should bear in mind that emissivity varies with temperature. All these measurement problems render the calibration process very important.

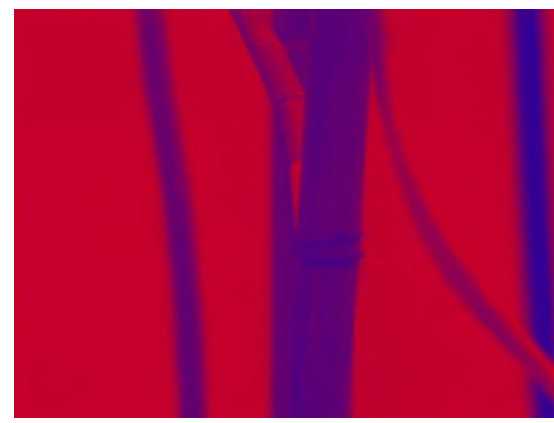

a)

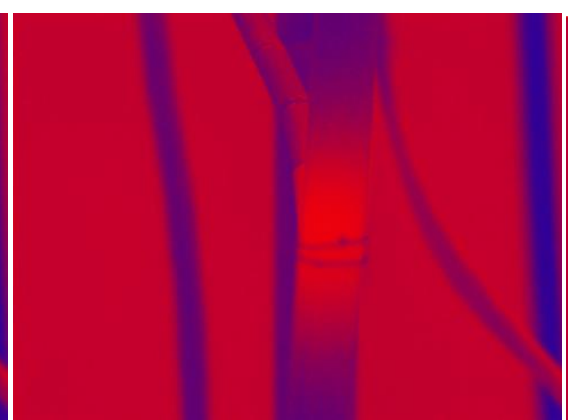

b)

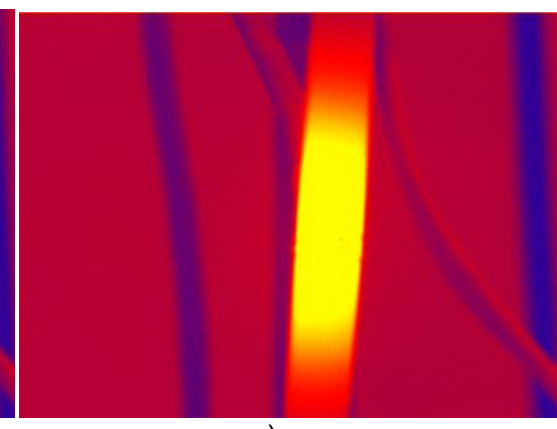

c)

Fig. 9. IR images for different temperature of the object, a) $300^{\circ} \mathrm{C}$; b) $400^{\circ} \mathrm{C}$; c) $500^{\circ} \mathrm{C}$

To confirm the correctness of the calibration performed, measurements of the temperature value in dynamic conditions during forced cooling of the reference object in a temperature range of $500^{\circ} \mathrm{C}-250^{\circ} \mathrm{C}$ were made. The studies were carried out in a dark room. The emissivity value of the reference object, determined previously, was taken into account - Table 2. During the measurement the integration time was automatically adjusted to the magnitude of radiation intensity of the object so as to avoid saturation of camera sensors. The result of the camera signal is presented in digital units $D L$ in fig. 10. Then, the $D L$ values were converted to temperature values according to calibration curve. At the same time, the temperature value was measured by means of an MWIR infrared camera. The results of temperature measurement with both NIR and MWIR cameras are presented in fig. 11.

The final experiment made at this stage of the research concerned the measurement of the tip of the soldering tool fig. 12. It was measured in the steady state using two systems for comparison: calibrated NIR and MWIR ones. The results were very coincident. The temperature of the tip was about $400^{\circ} \mathrm{C}$, and the disparity between the measurements made with NIR and MWIR were about $2^{\circ} \mathrm{C}$, i.e. $0.5 \%$.

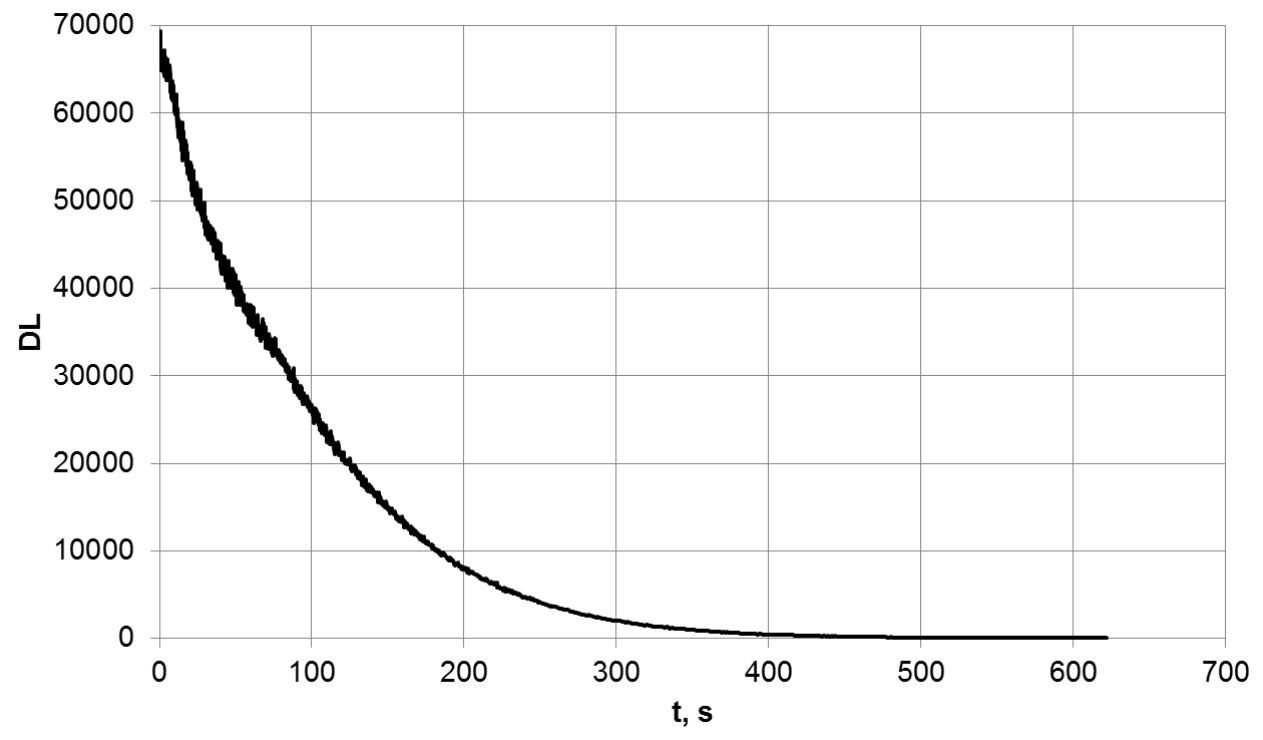

Fig.10. Curve of cooling of the reference object, camera signal in $D L$ units 


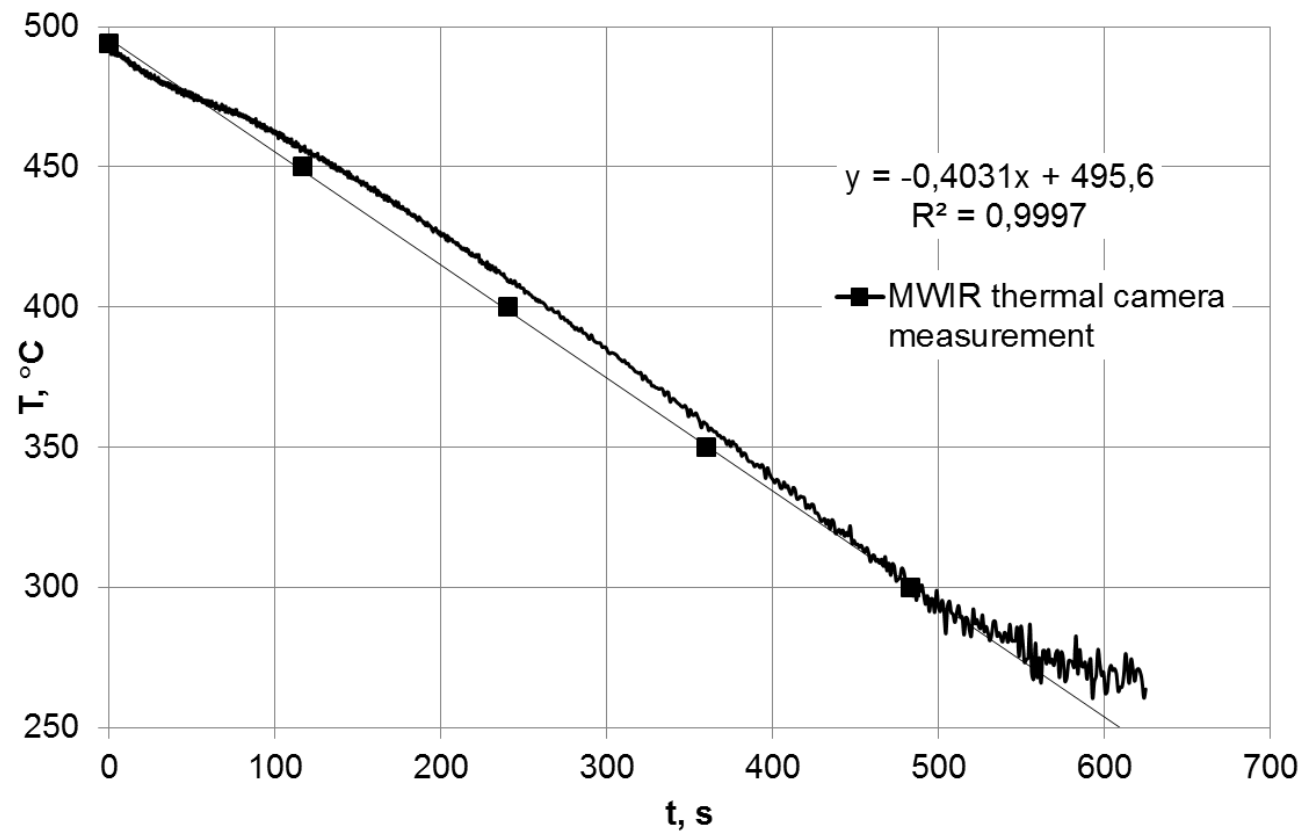

Fig. 11. Curve of cooling of the reference object, temperature in ${ }^{\circ} \mathrm{C}$

In Table 3 the results of temperature measurement and their relative error are presented. As can be seen, the error does not exceed $2.5 \%$.

Table 3. Comparison of temperature values measured with NIR and MWIR cameras

\begin{tabular}{|c|c|c|}
\hline $\begin{array}{c}\text { Temperature value measured } \\
\text { with NIR camera, }{ }^{\circ} \mathrm{C}\end{array}$ & $\begin{array}{c}\text { Temperature value measured with } \\
\text { MWIR camera, }{ }^{\circ} \mathrm{C}\end{array}$ & Relative error, \% \\
\hline 492.27 & 494 & -0.35 \\
\hline 456.39 & 450 & 1.42 \\
\hline 409.20 & 400 & 2.30 \\
\hline 358.15 & 350 & 2.33 \\
\hline 301.52 & 300 & 0.51 \\
\hline
\end{tabular}

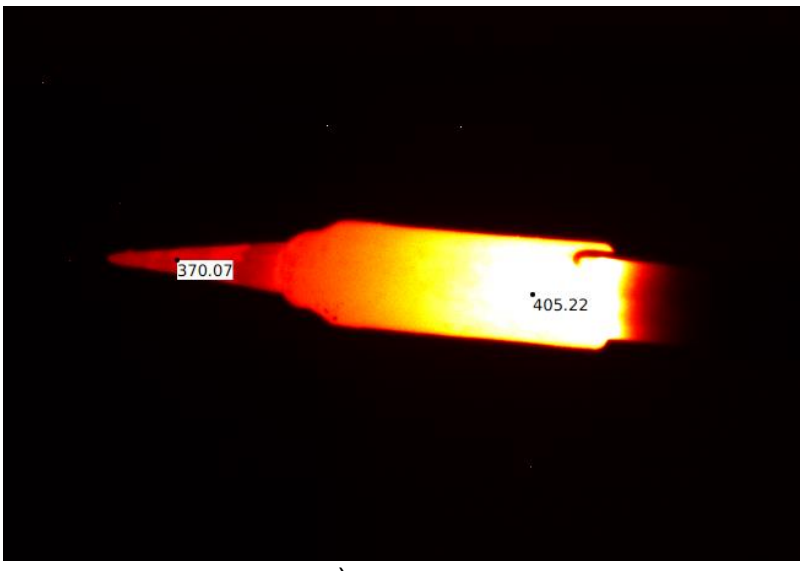

a)

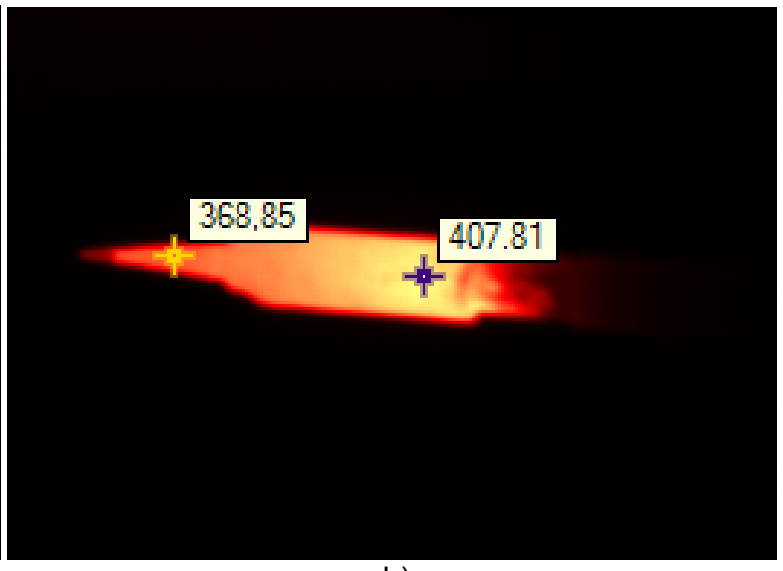

b)

Fig. 12. IR images of a soldering tool taken with the CCD-NIR camera (a) and MWIR thermal camera (b)

\section{Conclusions}

On the basis of the research carried out, the following comments and conclusions can be presented: 
- The application of dynamic correction of the integration time allows considerable extension of measurement range of the camera. Due to the linear relationship between the camera signal and the integration time, this correction can also be based on linear relationships. Without the adjustment of the integration time, the measurement would only be possible within a very small range of changes in the temperature value of the object under study.

- A calibration curve (in the work logarithmic approximation was used) can be determined on the basis of a number of measurement points, positioned mainly at a lower range of the temperatures measured, e.g. which, in the present work, ranged from 250 to $500^{\circ} \mathrm{C}$. In the absence of a black body for the NIR range analysed, it is possible to perform calibration e.g. using a black body for the medium wavelength infrared (MWIR).

- In the NIR range analysed, it is necessary to take into consideration the effect of emissivity of both the reference object used in the calibration process and the object studied during temperature measurement. To do so, a mirror for the near infrared spectrum (polished copper sheet).

In the present paper, preliminary results of the temperature measurement accuracy by the method of analysis of radiation in the NIR range have been presented and evaluated. For the evaluation of accuracy the simultaneous measurement of the temperature value in the NIR and MWIR range by means of an infrared camera.

\section{Acknowledgment}

This work was supported by the Polish National Centre for Research and Development (NCBiR) under Project PBS1/A4/2/2012

\section{REFERENCES}

[1] Jürgen Hartmann, New Developments in High-Temperature Measurement Techniques, International Journal of Electrical and Computer Engineering Systems, Volume 5, Number 2, 2014.

[2] Vladimir Firago, Waldemar Wojcik, High-temperature three-color thermal imager, Przegląd Elektrotechniczny, ISSN 0033-2097, R. 91 NR 2/2015.

[3] M. Hain, J. Bartl, V. Jacko, The use of infrared radiation in measurement and non-destructive testing, Measurement Science Review, Volume 5, Section 3, 2005.

[4] F. Meriaudeau, Real Time Multispectral High Temperature Measurement: Application to control in the industry Image and Vision Computing Volume 25, Issue 7, 1 July 2007.

[5] Strąkowski Robert, Pacholski Krzysztof, Więcek Bogusław, Olbrycht Robert, Wittchen Wacław, Borecki Mariusz: Radiative parameters of steel slag for $\mathrm{FeO}$ content estimation using multispectral thermography system. Quantitative InfraRed Thermography Journal, 2014, vol. 11, no. 2, p. 222-232, ISSN: 1768-6733.

[6] Fabijańska A., Sankowski D., Optical Filters Influence on Digital Image Quality in High Temperature Measurement of Surface Properties, Journal of Applied Computer Science Methods, vol. 1. No. 2, 2010, pp.53-63. 